\title{
Pacific
}

Journal of

Mathematics

\section{STUDYING LINKS VIA CLOSED BRAIDS. VI. A} NONFINITENESS THEOREM

\author{
JoAn Birman and WiLliam W. Menasco
}




\title{
STUDYING LINKS VIA CLOSED BRAIDS VI: A NON-FINITENESS THEOREM
}

\author{
Joan S. Birman and William W. Menasco
}

Exchange moves were introduced in an earlier paper by the same authors. They take one closed $n$-braid representative of a link to another, and can lead to examples where there are infinitely many conjugacy classes of $n$-braids representing a single link type.

THEOREM 1. If a link type has infinitely many conjugacy classes of closed $n$-braid representatives, then $n \geq 4$ and the infinitely many classes divide into finitely many equivalence classes under the equivalence relation generated by exchange moves.

This theorem is the last of the preliminary steps in the authors' program for the development of a calculus on links in $S^{3}$.

THEOREM 2. Choose integers $n, g \geq 1$. Then there are at most finitely many link types with braid index $n$ and genus $g$.

Introduction. This paper is the sixth in a series in which the authors study the closed braid representatives of an oriented link type $\mathscr{L}$ in oriented 3-space. The earlier papers in the series are [B-M,I]-[B$\mathbf{M}, \mathbf{V}]$. An overall view of the program may be found in [B-M]. The long-range goal of the program is to classify link types, up to isotopy in oriented 3-space, using techniques based upon the theory of braids. This paper is the last of the preliminary steps on the way to so doing.

Let $\mathscr{L}$ be an oriented link in oriented 3-space, and let $\mathbf{L}$ be a closed $n$-braid representative of $\mathscr{L}$, with braid axis $\mathbf{A}$. If the isotopy class of $\mathbf{L}$ in $S^{3}-\mathbf{A}$ has a representative which has the very special form illustrated in Figure 1 (see next page), then $\mathbf{L}$ is said to admit an exchange move, as illustrated in Figure 1. (The example shown there is a 4-braid; however if each strand is replaced by some number of parallel strands, it can be reinterpreted as an $n$ braid, for any $n$.) Exchange moves take closed $n$-braids to closed $n$-braids, in general changing the conjugacy class. Figure 2 (see next page) shows how $n$-braids which admit exchange moves may be modified to produce infinitely many closed $n$-braid representatives of $\mathscr{L}$. In effect, the exchange move allows one to replace the sub-braid $\mathbf{X}$ 


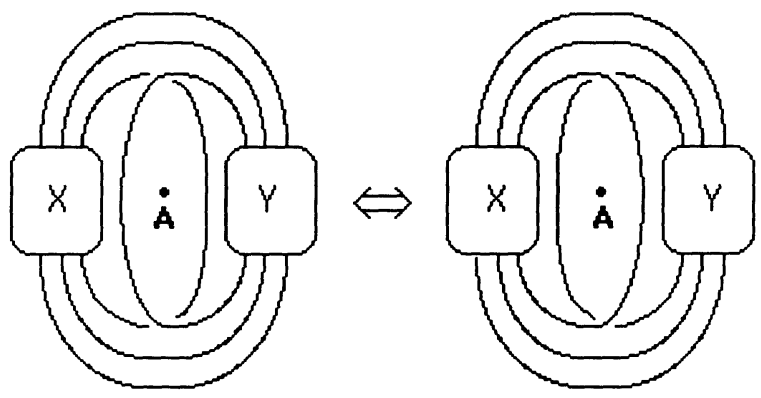

Exchange move

FIGURE 1

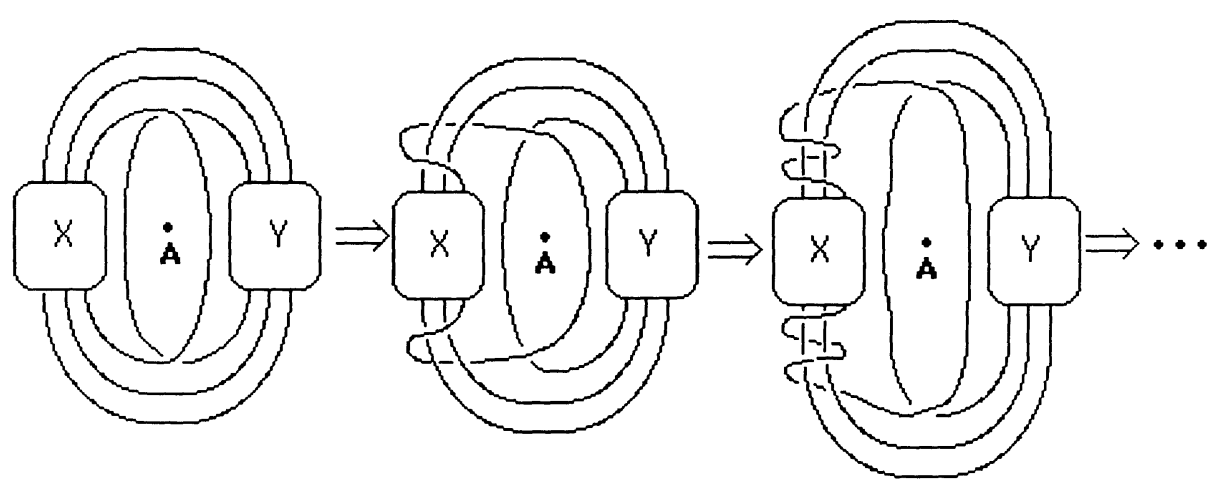

FIGURE 2

by a very special conjugate of itself, leaving $\mathbf{Y}$ invariant. For generic braids $\mathbf{X}$ and $\mathbf{Y}$ the infinitely many $n$-braids so produced will be in distinct conjugacy classes, because the isotopy which is shown is in general not realizable as an isotopy in the complement of $\mathbf{A}$. See [VB] for a proof of this assertion, for specific choices of $\mathbf{X}$ and $\mathbf{Y}$. To understand how this phenomenon can lead to serious complications in the $n$-braid representations of knots and links, recall that it was shown in [B-M,V] that there is a 4-braid representative of the unknot which admits an exchange move, and that by modifying it as in Figure 2 one obtains infinitely many distinct conjugacy classes of 4-braid representatives of the unknot. But then, any closed braid representative of any link may be connect-summed with this particular closed braid representative of the unknot, to produce infinitely many similar examples for every link type.

The main result in this paper is that in fact exchange moves are the only way to produce infinitely many distinct conjugacy classes of closed $n$-braid representatives of a link type. 
THeOREM 1. Assume that $\mathscr{L}$ admits infinitely many conjugacy classes of $n$-braid representatives. Then $n \geq 4$, and the infinitely many conjugacy classes divide into finitely many equivalence classes under the equivalence relation generated by exchange moves.

As a corollary to Theorem 1, we will be able to say something about the exponent sum of a minimum-string braid representative of a link. Our corollary relates to

The Jones Conjecture. Let $\mathscr{L}$ be a link of braid index $n$, and let $\mathbf{L}$ and $\mathbf{L}^{\prime}$ be any two $n$-braid representatives. Express $\mathbf{L}$ and $\mathbf{L}^{\prime}$ in any way as words $\mathbf{W}$ and $\mathbf{W}^{\prime}$ in the elementary braids $\left(\sigma_{i}\right)^{ \pm 1}$. Then the exponent sums of $\mathbf{W}$ and $\mathbf{W}^{\prime}$ coincide.

By the work of Vaughan Jones $[\mathbf{J}]$ this conjecture is known to be true for $n=3$ and 4 . Our contribution is a weak version of the Jones Conjecture, which follows directly from Theorem 1.

Corollary. Let $\mathscr{L}$ be a link of braid index $n$. Then, among the $n$-braid representatives of $\mathscr{L}$, at most finitely many exponent sums can occur.

The referee has observed that there is another result which follows from the proof of Theorem 1:

Theorem 2. Choose integers $n, g \geq 1$. Then there are at most finitely many link types with braid index $n$ and genus $g$.

Most of this paper will be devoted to the proof of Theorem 1. At the very end, after we have completed our proof of Theorem 1, we will prove the Corollary and Theorem 2 .

Acknowledgment. We thank the referee for the detailed attention which he or she gave to our work, and for pointing out Theorem 2 to us.

Proof of Theorem 1. By hypothesis we are given a link type $\mathscr{L}$ which has infinitely many conjugacy classes $\left\{\left[\mathbf{L}_{i}\right] ; i=1,2,3, \ldots\right\}$ of $n$-braid representatives. We will first show that all but finitely many of the conjugacy classes $\left\{\left[\mathbf{L}_{i}\right] ; i=1,2,3, \ldots\right\}$ admit an exchange move. We will then show that this implies the stronger assertion. 
We may assume that $n \geq 4$. For, clearly $n \neq 1$, because the unknot is the only link of braid index 1. Also, $n \geq 2$, because $B_{2}$ is infinite cyclic, and since we are considering oriented links, each type $(2, p)$ torus link has a unique conjugacy class of 2-braid representatives, whereas the unknot has exactly 2 . The case $n=3$ is non-trivial; however it is proved in [B-M,III] that a link of braid index 3 (respectively 2,1 ) has 1 or 2 (respectively 2,3 ) conjugacy classes of 3-braid representatives. So $n \geq 4$.

Let $\mathbf{L}=\mathbf{L}_{1}$. Assume that $\mathbf{L}$ is the boundary of a not necessarily connected surface $\mathbf{F}$ which is oriented so that the positive normal bundle to $\mathbf{F}$ has the orientation induced by that on $\mathbf{L}$. Assume that F has been chosen to have maximum Euler characteristic $\chi$ among all such oriented spanning surfaces. A link $\mathbf{L}$ will in general have a multiplicity of such spanning surfaces; however having selected one we will stick with it throughout this paper. Thus each link $\mathbf{L}_{i}$ in our sequence will be assumed to be the boundary of $\mathbf{F}_{i}$, where if $i \neq k$ there is a homeomorphism $h_{i k}$ of $S^{3}$ such that $h_{i k}\left(\mathbf{F}_{k}\right)=\mathbf{F}_{i}$. In general $h_{i k}$ will not fix the braid axis $\mathbf{A}$, because by hypothesis $\mathbf{L}_{i}=\partial \mathbf{F}_{i}$ is in a different conjugacy class from $\mathbf{L}_{k}=\partial \mathbf{F}_{k}$.

The complement of the braid axis $\mathbf{A}$ in $R^{3}$ is an open cylinder, which is fibered by half-planes $\mathbf{H}_{\theta}$ through $\mathbf{A}$. Let $\mathbf{H}$ denote a choice of this fibration. The proof begins with a study of the foliation which is induced on $\mathbf{F}_{i}$ by its intersections with the fibers $\mathbf{H}_{\theta}$ of $\mathbf{H}$. This foliation was first studied by $\mathrm{D}$. Bennequin in [Be], in connection with his studies of contact structures on $R^{3}$. It was proved by Bennequin that $\mathbf{F}_{i}$ may be assumed to be a Markov surface. The first two properties of these surfaces are achieved by general position techniques:

(Mi) $\mathbf{A}$ intersects $\mathbf{F}_{i}$ transversally in a finite set of points $p_{1}, \ldots, p_{k}$ which we will refer to as "vertices." There is a neighborhood on $\mathbf{F}_{i}$ of each vertex which is foliated radially.

(Mii) All but finitely many fibers $\mathbf{H}_{\theta}$ of $\mathbf{H}$ meet $\mathbf{F}_{i}$ transversally, and those which do not (the singular fibers) are each tangent to $\mathbf{F}_{i}$ at exactly one point in the interior of both $\mathbf{F}_{i}$ and $\mathbf{H}_{\theta}$. Moreover, the tangencies are assumed to be either local maxima or minima or saddle points.

A third property is obtained by Bennequin [Be] by small modifications in the $\mathbf{F}_{i}$ 's. A proof is also given in Lemma 2 of [B-M,I]:

(Miii) There are no simple closed curves in the foliation of $\mathbf{F}_{i}$. If $\mathbf{H}_{\theta}$ is a non-singular fiber, then each component of $\mathbf{H}_{\theta} \cap \mathbf{F}_{i}$ is an arc. 
Following [B-M,I], pairs $(\mathbf{F}, \mathbf{H})$ and $\left(\mathbf{F}^{\prime}, \mathbf{H}^{\prime}\right)$ which satisfy (Mi), (Mii) and (Miii) will be said to be equivalent if there is an isotopy $h_{t}$ of $R^{3}, t \in[0,1]$, which takes $(\partial \mathbf{F}, \partial \mathbf{H})$ to $\left(\partial \mathbf{F}^{\prime}, \partial \mathbf{H}^{\prime}\right)$ in such a way that each $h_{t}(\mathbf{F}, \mathbf{H})$ satisfies $(\mathbf{M i})$, (Mii) and (Miii). Let $[\mathbf{F}, \mathbf{H}]$ be the equivalence class of $(\mathbf{F}, \mathbf{H})$. Let $|\mathbf{A} \cap \mathbf{F}|$ denote the number of points of intersection of $\mathbf{A}$ with $\mathbf{F}$. Let $|\mathbf{H} \cdot \mathbf{F}|$ be the number of tangencies between $\mathbf{F}$ and fibers of $\mathbf{H}$. The complexity $C([\mathbf{F}, \mathbf{H}])$ is defined to be the pair $(|\mathbf{A} \cap \mathbf{F}|,|\mathbf{H} \cdot \mathbf{F}|)$. It is well-defined on equivalence classes.

We continue to investigate $\mathbf{F}_{i}$. The next property, established in Lemma 1 of $[\mathbf{B}-\mathbf{M}, \mathbf{I}]$, follows from the fact that $\mathbf{L}_{i}=\partial \mathbf{F}_{i}$ is a closed braid, so that all its intersections with fibers of $\mathbf{H}$ are coherently oriented:

(Miv) An arc of intersection of $\mathbf{F}_{i}$ with a non-singular fiber of $\mathbf{H}$ never has both of its endpoints on $\mathbf{L}_{i}$.

It follows from (Miv) that the arcs in $\mathbf{F}_{i} \cap \mathbf{H}_{\theta}$ for non-singular $\mathbf{H}_{\theta}$ are restricted to two types:

a-arcs: one endpoint is on $\mathbf{A}$ and the other is on $\mathbf{L}_{i}$,

b-arcs: both endpoints are on $\mathbf{A}$,

and that the singularities are restricted to three types:

aa (if the arcs which come together are both type a), bb (if the arcs which come together are both type b), ab (if one is type a and the other type b).

A b-arc $\beta$ in $\mathbf{H}_{\theta} \cap \mathbf{F}_{i}$ is essential if both sides of $\mathbf{H}_{\theta}$ split along $\beta$ are pierced by $\mathbf{L}$. If $\beta$ is inessential, then $\beta$ and a subarc $\alpha$ of $\mathbf{A}$ will co-bound a disc $\mathbf{D}$ on $\mathbf{H}_{\theta}$ which is not pierced by $\mathbf{L}_{i}$. The axis $\mathbf{A}$ may then be pushed across $\mathbf{D}$ to eliminate two points of $\mathbf{A} \cap \mathbf{F}_{i}$, and so to reduce the number of points in $\mathbf{A} \cap \mathbf{F}_{i}$. After the reduction, one may recover (Mi)-(Miv) without increasing the complexity (see [B-M,IV] for details), so from now on we may assume:

(Mv) Every b-arc in the foliation is essential.

Finally, a component of $\mathbf{F}_{i}$ is trivially foliated if $\mathbf{F}_{i}$ is a disc which is pierced once by $\mathbf{A}$, and if $\mathbf{F}_{i}$ is radially foliated by its arcs of intersection with fibers of $\mathbf{H}$. Since the boundary of a trivially foliated component is necessarily a 1-braid representative of the unknot, a case which is not of interest in this paper, we assume from now on that:

(Mvi) No component of $\mathbf{F}_{i}$ is trivially foliated. 
We begin to study the combinatorics of the foliation of $\mathbf{F}_{i}$. To simplify the notation we will assume (until near the end of the proof) that $\mathbf{F}_{i}$ is connected. Later we will show how to modify things if $\mathbf{F}_{i}$ is disconnected. As in [B-M,I] we may choose a finite collection $\mathscr{E}_{i}$ of a-arcs and b-arcs such that $\mathbf{F}_{i}$ split along $\mathscr{E}_{i}$ is a union of foliated 2-cells $\mathscr{T}_{i}$, which we call tiles. See Figure 3. The arcs in $\mathscr{E}_{i}$ are chosen so that there is exactly one singularity of the foliation in the interior of each tile. The left column in Figure 3 shows the three types of tiles which occur. The tile edges are the arcs in $\mathscr{E}_{i}$, shown as dotted lines. The singular leaves as solid lines, and subarcs of $\mathbf{L}$ are thick solid lines. The singularities are indicated by black dots. The tile vertices $\mathscr{V}_{i}$ are the points where $\mathbf{A}$ pierces $\mathbf{F}_{i}$. They are labeled $p_{1}, p_{2}, \ldots$. We have also labeled the L-endpoints of the singular leaves with symbols $\lambda_{1}, \lambda_{2}, \ldots$. The tiles are denoted as being types $\mathbf{a a}, \mathbf{a b}$ and $\mathbf{b b}$, according as the singularity is type aa, ab or bb. There are four a-arcs in the boundary of an aa-tile and four b-arcs in the boundary of a bb-tile, and two of each type in the boundary of an ab-tile.

For future use, we record at this time two other features of the combinatorics which we will use in this paper. The foliation of $\mathbf{F}_{i}$ was assumed to be radial about each vertex, so as we push forward in the positive direction through the fibration, the arcs of intersection of $\mathbf{F}_{i}$ with fibers of $\mathbf{H}$ will flow radially about the vertices. This flow will be anticlockwise or clockwise, according as the oriented axis $\mathbf{A}$ intersects $\mathbf{F}_{i}$ from the positive or from the negative side. Call a vertex positive in the former case and negative in the latter. Notice that the braid index is the number of positive vertices minus the number of negative vertices.

The type of a vertex is the cyclically ordered array of a's and b's which records the tile edges meeting at that vertex. We shall not need it here as an oriented symbol, but we might as well orient it by the flow about the vertex. Later we will need the fact (see Figure 3) that the flow is always positive about a vertex which contains an a in its type symbol. On the other hand, a vertex of type $\mathbf{b b} \cdots \mathbf{b}$ could be either positive or negative. The valence of a vertex is the number of symbols in its type symbol.

In the right column of Figure 3 we have shown how the singular leaves would look if they are viewed on the singular fiber $\mathbf{H}_{\theta}$ of $\mathbf{H}$. We have used the same cyclic order for the four endpoints of the singular leaves in the left and right columns; this means that the view 


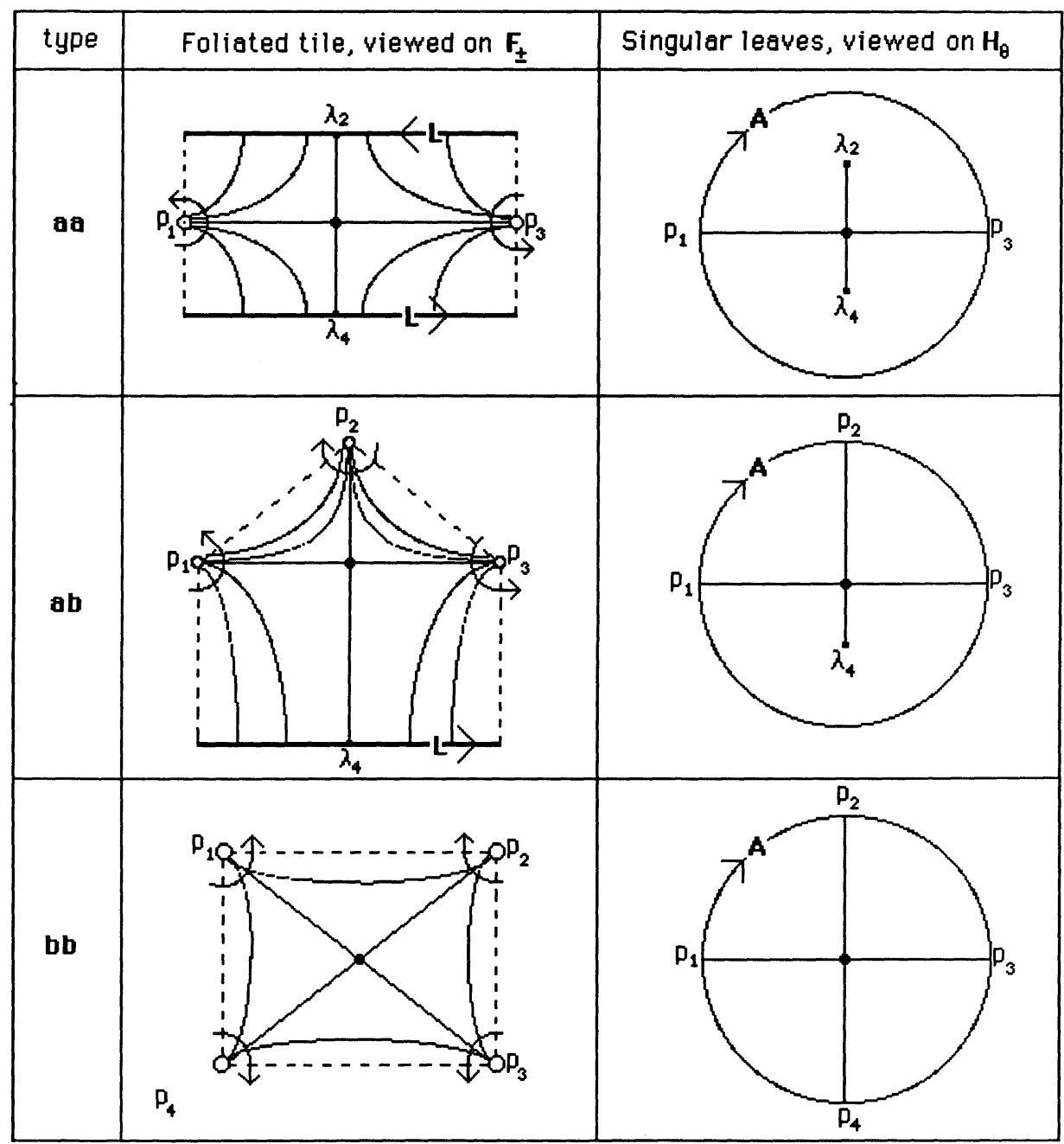

\section{Figure 3}

on $\mathbf{F}_{i}$ must be interpreted as being on the positive or negative side of $\mathbf{F}_{i}$, according as the oriented normal to $\mathbf{F}$ at the singularity points in the direction of increasing or decreasing $\theta$. Figure 4 (see next page) gives an example to indicate to the reader how distinct tile types might fit together, in the case where $\mathbf{F}_{i}$ is a disc. (This example can be realized by the unknot, represented as a 2-braid $\mathbf{L}$.)

Recall that $\mathscr{V}_{i}, \mathscr{E}_{i}$ and $\mathscr{T}_{i}$ are the sets of tile vertices, edges and tiles in the tiling of $\mathbf{F}_{i}$. Notice that our method of counting is rather special. We do not count subarcs of $\mathbf{L}_{i}$ which are in the boundary of a tile as an edge. Thus each tile has exactly four "edges." Similarly, we do not include in $\mathscr{V}_{i}$ the points where a tile edge meets $\mathbf{L}_{i}$. Let 


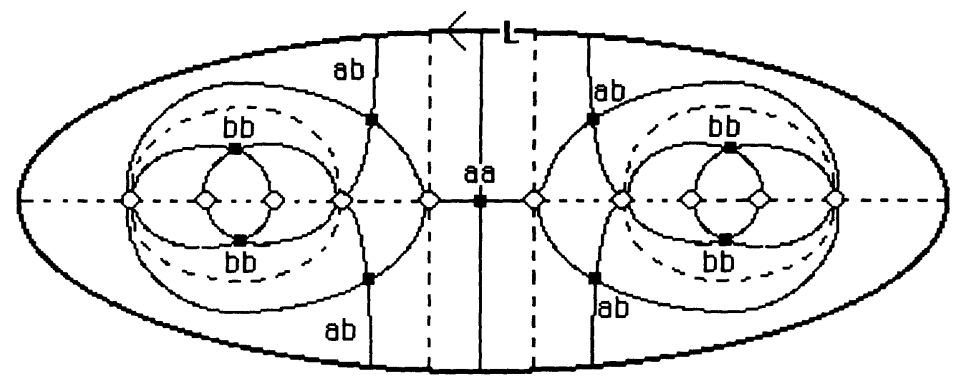

Example: a tiling of a disc with no vertices of valence 1

FIGURE 4

$V_{i}, E_{i}$ and $T_{i}$ be the cardinality of $\mathscr{V}_{i}, \mathscr{E}_{i}$ and $\mathscr{T}_{i}$ respectively. The next two lemmas relate to results established by the authors in other papers of this series.

LEMma 3. If the tiling of $\mathbf{F}_{i}$ has a vertex of valence 1 , then $\mathbf{L}_{i}$ admits an exchange move.

Proof. It is proved in Lemma 5 of $[\mathbf{B}-\mathbf{M}, \mathbf{V}]$ that if there is a vertex of valence 1 , then $\mathbf{L}_{i}$ has a trivial loop. But then, as is shown in Figure 5, we may reconfigure the closed braid so that $\mathbf{L}_{i}$ is seen to admit an exchange move.

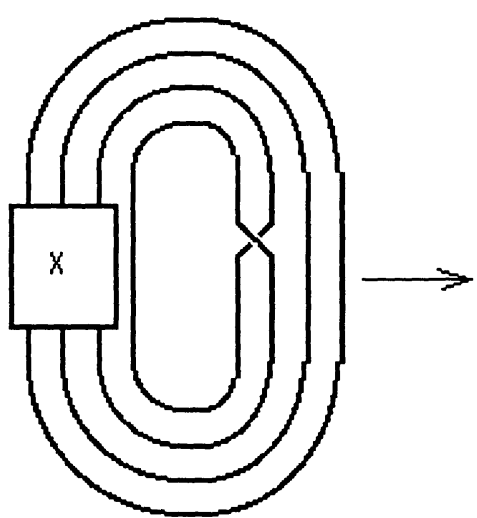

closed braid which has a trivial loop

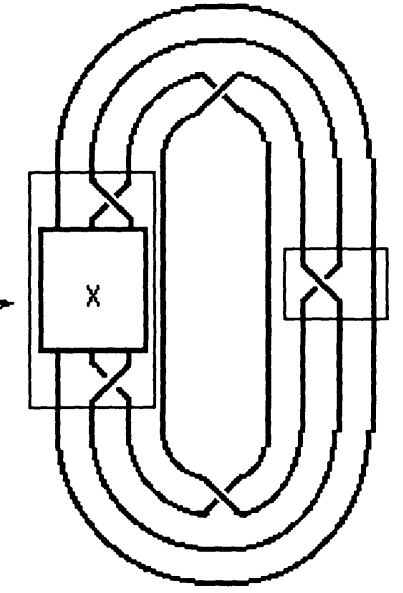

the same closed braid, re-configured so it admits exchange mave 
Since our initial goal was to show that all but finitely many $\mathbf{L}_{i}$ admit an exchange, we will have achieved our goal if there is a vertex of valence 1 in the tiling of $\mathbf{F}_{i}$. Therefore we may assume from now on that the tiling of $\mathbf{F}_{i}$ has no vertices of valence 1. Let $V_{i}(\alpha, \beta)$ denote the number of vertices in the tiling of $\mathbf{F}_{i}$ which have $\alpha$ a-arcs and $\beta$ b-arcs in their type symbols.

LEMMA 4. If any one of $V_{i}(1,1)$ or $V_{i}(0,2)$ or $V_{i}(0,3)$ is nonzero, then $\mathbf{L}_{i}$ admits a complexity-reducing exchange move.

Proof. It was proved in Lemma 5 of [B-M,IV] that if there is a vertex of type $\mathbf{b b}$ in the tiling of $\mathbf{F}_{i}$, then $\mathbf{L}_{i}=\partial \mathbf{F}_{i}$ admits a complexityreducing exchange. The corresponding statement for vertices of type ab is proved in Lemma 4 of [B-M,V], when the array of signs of the singularities in the tiles which meet at the vertex are (+-). The case of vertices of type bbb is reduced to the case of vertices of type bb by Lemma 8 of $[\mathbf{B}-\mathbf{M}, \mathbf{V}]$. Type $\mathbf{a b}$, with sign sequence $(++)$ or $(--)$, is reduced to the case where there is a vertex of valance 1 by Lemma 9 of [B-M,V]. (Remark: this sign sequence is independent of the sign of a vertex, as we defined it earlier.) Thus, in every case $\mathbf{L}_{i}$ admits a complexity-reducing exchange.

We now proceed to analyze the consequences of our hypothesis that we have an infinite sequence $\left\{\mathbf{L}_{i} ; i \in N\right\}$ of closed $n$-braid representatives of $\mathscr{L}$, with $\mathbf{L}_{i}$ in a different conjugacy class from $\mathbf{L}_{k}$ if $i \neq k$. We look to the tiling of $\mathbf{F}_{i}$ for information about the existence of exchange moves.

LEMMA 5. The number $V_{i+}$ of positive vertices in the tiling of $\mathbf{F}_{i}$ and also the number $V_{i-}$ of negative vertices goes to infinity as $i \rightarrow \infty$, with $V_{i+}-V_{i-}$ remaining constant.

Proof. The surface $\mathbf{F}_{i}$ admits a non-trivial tiling, relative to our particular choice of fibration $\mathbf{H}$ of $S^{3}-\mathbf{A}$. We now use the tiling to make an Euler characteristic count. Since there are no vertices of valence 1 , it follows that if one shrinks $\partial \mathbf{F}_{i}$ to a point, the image of each tile under the collapsing map will be a 2-cell with 4 edges and 4 vertices. Thus:

$$
2 T_{i}=E_{i} .
$$

The collapsing map increases the number of tile vertices by 1 , but it also raises the Euler characteristic of $\mathbf{F}_{i}$ by 1 . The two effects cancel, 
so we also have:

$$
V_{i}-T_{i}=\chi
$$

Recall that the complexity of $\left[\mathbf{F}_{i}, \mathbf{H}\right]$ is the pair $\left(V_{i}, T_{i}\right)$, or equivalently (in view of equation (2)) the pair $\left(V_{i}, V_{i}-\chi\right)$. It was proved in Theorem 2 of [B-M,I] that there are at most finitely many equivalence classes $\left[\mathbf{F}_{i}, \mathbf{H}\right]$ for each fixed value of the complexity. This means that there are at most finitely many distinct conjugacy classes $\left[\mathbf{L}_{i}\right]$ for each fixed value of the complexity. Since $\chi$ is fixed, and since by hypothesis infinitely many distinct conjugacy classes $\left[\mathbf{L}_{i}\right]$ occur, the only possibility is that $V_{i}$ goes to infinity as $i \rightarrow \infty$.

Now recall that $V_{i}=V_{i+}+V_{i-}$ and $V_{i+}-V_{i-}=n$, the braid index. Since $n$ is by hypothesis independent of $i$, the assertion follows.

We continue our proof of Theorem 1. The symbol $V_{i}(\alpha, v-\alpha)$ denotes the number of vertices in the tiling of $\mathbf{F}_{i}$ which have valence $v$ and have $\alpha$ a-arcs as edges. Thus, we can express $V_{i}$ as the following sum of the $V_{i}(\alpha, v-\alpha)$ 's:

$$
V_{i}=\sum_{v=2}^{\infty} \sum_{\alpha=0}^{v} V_{i}(\alpha, v-\alpha) .
$$

Let $E_{i}(a)$ and $E_{i}(b)$ denote the number of type $\mathbf{a}$ and $\mathbf{b}$ edges, respectively, in the tiling, so that $E_{i}=E_{i}(a)+E_{i}(b)$. Since each type a edge is incident at one vertex, whereas each type b edge is incident at two vertices, we have related sums $E_{i}(a)$ and $E_{i}(b)$ :

$$
\begin{gathered}
E_{i}(a)=\sum_{v=2}^{\infty} \sum_{\alpha=0}^{v} \alpha V_{i}(\alpha, v-\alpha), \\
2 E_{i}(b)=\sum_{v=2}^{\infty} \sum_{\alpha=0}^{v}(v-\alpha) V_{i}(\alpha, v-\alpha) .
\end{gathered}
$$

Using equation (1) to rewrite equation (2) in the form $4 V_{i}-2 E_{i}(a)-$ $2 E_{i}(b)=4 \chi$, we may then combine it with equations (3), (4) and (5) to obtain:

$$
4 \chi=\sum_{v=2}^{\infty} \sum_{\alpha=0}^{v}(4-v-\alpha) V_{i}(\alpha, v-\alpha)
$$

Note that when $v \geq 4$ the coefficient $(4-v-\alpha)$ will be non-positive. We may thus alter equation (6) so that all terms on both sides of the 
equation, except possibly $4 \chi$, are non-negative. This gives:

$$
\begin{aligned}
& V_{i}(1,1)+2 V_{i}(0,2)+V_{i}(0,3) \\
& =4 \chi+V_{i}(2,1)+2 V_{i}(3,0) \\
& \quad+\sum_{v=4}^{\infty} \sum_{\alpha=0}^{v}(v+\alpha-4) V_{i}(\alpha, v-\alpha) .
\end{aligned}
$$

Recall that the type of a vertex $p$ in the tiling of $\mathbf{F}_{i}$ was defined to be the cyclic array of a's and b's which records the types of the tile edges which meet at $p$. Thus if $V_{i}(1,1) \neq 0$ (respectively $V_{i}(0,2) \neq$ $0, V_{i}(0,3) \neq 0$ ) there is a vertex of type ab (respectively $\mathbf{b b}, \mathbf{b b b}$ ) in the tiling of $\mathbf{F}_{i}$.

We now consider the possible ways in which $V_{i} \rightarrow \infty$. Equation (7) will record any change in the differing values of the $V_{i}(\alpha, v-\alpha)$ 's, except for the cases $(\alpha, v-\alpha)=(1,2),(2,0)$ and $(0,4)$, since in those cases the coefficient $(v+\alpha-4)$ which occurred in equation (6) was zero, so that the corresponding terms do not appear in equation (7). For all other values of $(\alpha, v-\alpha)$ we see that if $V_{i}(\alpha, v-\alpha)$ increases without bound the left-hand side of equation (7) would be forced to be non-zero. Thus there are two possibilities:

possibility 1:

At least one of $V_{i}(1,1), V_{i}(0,2)$ or $V_{i}(0,3) \neq 0$. possibility 2:

$V_{i}(1,1)=V_{i}(0,2)=V_{i}(0,3)=0$ for all $i$, also at least one of the following holds:

$$
\begin{aligned}
& V_{i}(1,2) \rightarrow \infty \quad \text { as } i \rightarrow \infty, \\
& V_{i}(2,0) \rightarrow \infty \quad \text { as } i \rightarrow \infty, \\
& V_{i}(0,4) \rightarrow \infty \quad \text { as } i \rightarrow \infty,
\end{aligned}
$$

and also (passing to a subsequence if necessary) $V_{i}(\alpha, \beta)$ is independent of $i$ for all other $(\alpha, \beta)$.

If possibility 1 occurs, then by Lemma 4 we conclude that $\mathbf{L}_{i}$ admits an exchange move. Thus we are reduced to possibility 2 .

LEMMA 6. In the situation of possibility 2 , the cases $V_{i}(2,0) \rightarrow \infty$ and $V_{i}(1,2) \rightarrow \infty$ do not occur.

Proof of Lemma 6. We first show that $V_{i}(2,0) \rightarrow \infty$ is impossible, i.e. that the number of vertices of type aa cannot grow without bound as $i \rightarrow \infty$. Such a vertex can only occur when two aa tiles are joined 


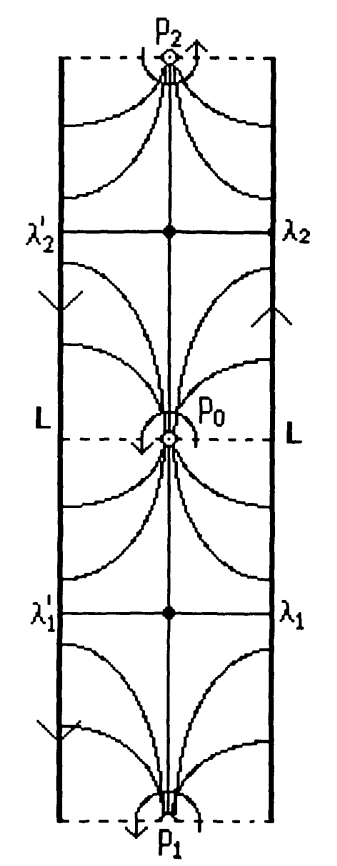

$P_{0}$ is a vertex of type $\mathbf{a a}$

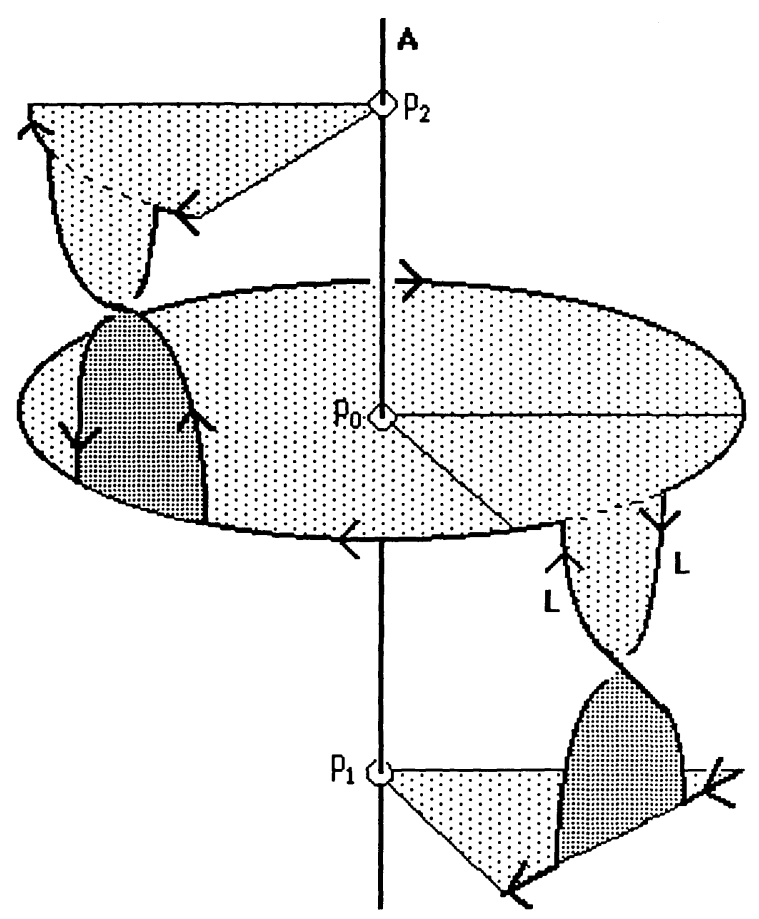

Figure 6

together along a pair of common edges. We need to see how this situation would look in 3-space, and to do so we ask how two aa tiles appear imbedded in 3-space. The left picture in Figure 6 shows two aa tiles glued together along two common a-edges, to give a vertex of type aa. We focus first on the tile with vertices $p_{0}$ and $p_{1}$. Its singular leaves lie on some fiber $\mathbf{H}_{\theta}$ of $\mathbf{H}$, with one of them having its endpoints at points $p_{0}$ and $p_{1}$ on the axis $\mathbf{A}$, and the other having its endpoints at $\lambda$ and $\lambda^{\prime}$ on $\mathbf{L}_{i} \cap \mathbf{H}_{\theta}$. The right picture in Figure 6 shows these leaves in 3-space (to be imagined as lying on a single fiber). Since the embedded tile is transverse to the fibers everywhere else, the final picture (which is determined up to reversal of the halftwist in the band) must be as in the right picture in Figure 6 . The second tile will have a similar imbedding (ambiguous up to the choice of the order of the three vertices on $\mathbf{A}$ and the senses of the halftwists), but in all cases we see that if $V_{i}(2,0)$ were to grow without bound the braid index $n$ would too, contrary to hypothesis.

Suppose next that $V_{i}(1,2) \rightarrow \infty$ as $i \rightarrow \infty$, i.e. the number of vertices of type abb grows without bound. Let $p$ be a vertex of type 

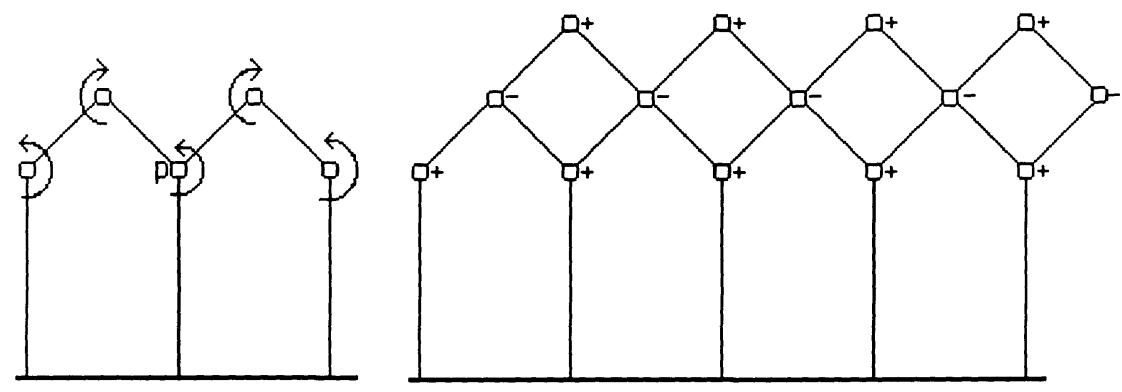

FIGURE 7

abb. Then $p$ is positive, and the a edge which is incident at $p$ must be on an ab tile. Thus there are necessarily two ab tiles glued together along a common a-edge which meet $p$. We can think of them as a pair of attached houses, as in the left picture of Figure 7. Since the unique vertex which is at the peak of the roof in an ab tile is a negative vertex, we cannot glue an ab tile into the trough. So the only way to fill in the trough is with a bb tile, as illustrated. So, if the number of vertices of type abb goes to infinity as $i \rightarrow \infty$, we will necessarily find tiles in our sequence with arbitrarily long rows of attached houses glued together in a row as in the right picture in Figure 7 , with a bb tile in the trough between each pair of adjacent roofs.

We now investigate the contributions to $V_{i+}$ from our row of houses. The sum of the signs in each bb tile is zero, but the sum of the signs in each ab tile is +1 . If our sequence contained $k_{i}$ tiles of type ab, we would also have $k_{i}$ tiles of type bb, and so there would be a net partial contribution of $k_{i}$ to $V_{i+}$. Since $\left(V_{i+}-V_{i-}\right)$ must be independent of $i$, this means that we will need to have $k_{i}$ extra negative vertices somewhere else. However, that is impossible, because the sum of the signs of the vertices in our 3 tile types is $+2,+1$ and 0 . Thus, if $V_{i}(1,2) \rightarrow \infty$ as $i \rightarrow \infty$, the braid index will be forced to increase without bound, contrary to hypothesis.

Possibility 2 has been reduced to the situation where $V_{i}(0,4) \rightarrow \infty$ as $i \rightarrow \infty$. Passing to a subsequence if necessary, we may assume that $V_{i}(1,2)$ and $V_{i}(2,0)$ are independent of $i$.

LemMA 7. Allowing $V_{i}(0,4)$ to go to $\infty$ as $i \rightarrow \infty$ only gives finitely many distinct conjugacy classes of closed $n$-braids representing our link $\mathscr{L}$. 


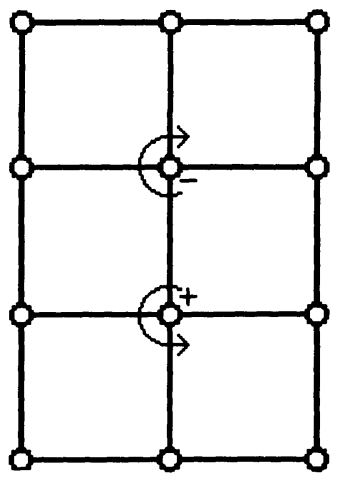

Figure 8

Proof of Lemma 7. Assume that $V_{i}(0,4) \rightarrow \infty$ as $i \rightarrow \infty$. By Lemma 5 the number of positive vertices of type bbbb and also the number of negative vertices of type bbbb must go to infinity as $i \rightarrow \infty$, with $V_{i+}-V_{i-}$ remaining constant. From Figure 3 we see that the only tile type which contains a positive vertex at which $\mathbf{b}$-arcs are incident is a type bb tile, so the only way to obtain a positive vertex of type bbbb is as in Figure 8. So this picture occurs infinitely often.

Now notice that the sum of the signs of the vertices in a bb tile is zero. It then follows that the negative vertices of type bbbb must also lie at the intersection of four bb tiles, because if there were infinitely many such negative vertices which included an ab tile, the difference $V_{i+}-V_{i-}$ would not remain constant. Thus there must be interior regions of $\mathbf{F}_{i}$ which are tiled entirely by bb tiles. Moreover (passing to a subsequence if necessary) we may assume that the tiling remains fixed outside one such region, whereas the number of bb tiles in the region increases without bound as $i$ is increased. Since the Euler characteristic is independent of $i$, the region in question must be an annulus. The tiling on the boundary of our annulus must be fixed, so the only way things can change is if the number of tiles between the two boundary components grows without bound as $i \rightarrow \infty$. See Figure 9 for an example of one way this could occur. The difference $V_{i+}-V_{i-}$ will thus remain independent of tube length, as will the Eulẹr characteristic of $\mathbf{F}_{i}$.

Recall our assumption that $V_{i}(\alpha, \beta)$ is independent of $i$ if $(\alpha, \beta) \neq$ $(0,4)$. Even more, by passing to a subsequence if necessary, we may assume that the tiling of $\mathbf{F}_{i}$ is fixed in the component of our annulus. 

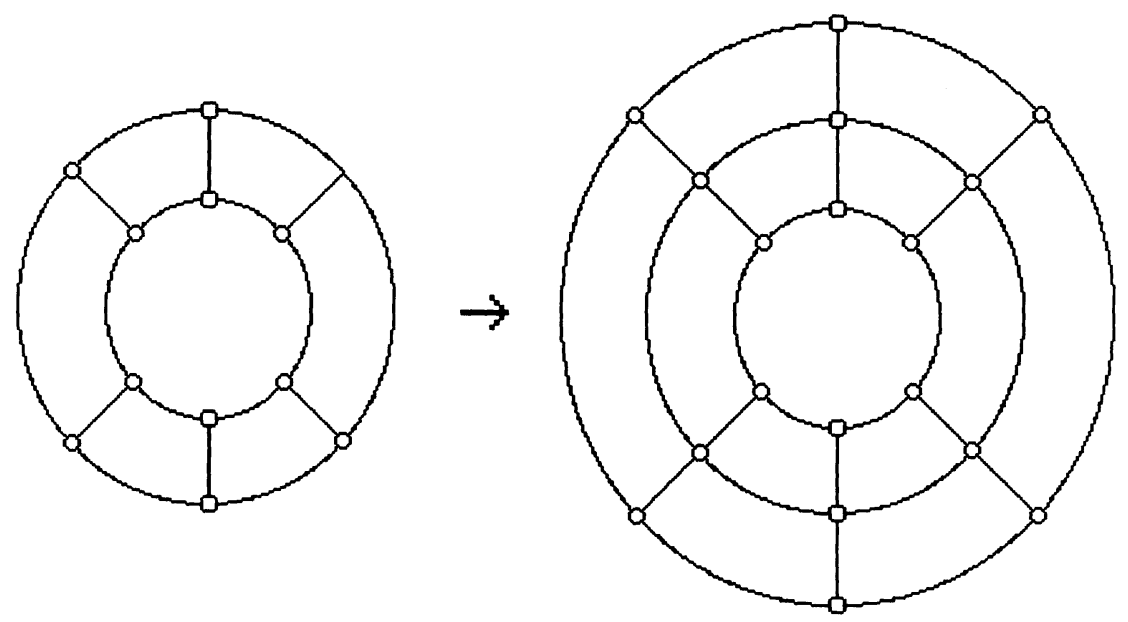

FIGURE 9

An additional property then follows immediately from equation (4):

$$
E_{i}(a) \text { is independent of } i \text {. }
$$

Let $T_{i}(a a)$ and $T_{i}(a b)$ denote the number of tiles of type aa and $\mathbf{a b}$ in the tiling of $\mathbf{F}_{i}$. Notice that a tile of type aa (respectively $\mathbf{a b}$ ) contains 4 (respectively 2 ) edges of type a. Since each edge is an edge of two tiles, we then have:

$$
E_{i}(a)=2 T_{i}(a a)+T_{i}(a b) .
$$

Since $E_{i}(a)$ is independent of $i$, by $(8)$, we conclude that $2 T_{i}(a a)+$ $T_{i}(a b)$ is too. Thus

$$
\begin{aligned}
& T_{i}(a a) \text { is independent of } i, \\
& T_{i}(a b) \text { is independent of } i .
\end{aligned}
$$

It will be convenient now to assume that $\mathscr{L}$ is a knot. Later we will modify the proof to the case where $\mathscr{L}$ is a link. Our idea is to show that a finite set of combinatorial data in the tiling of $\mathbf{F}_{i}$ determines $\mathbf{L}_{i}$ as an embedded simple closed curve, relative to $\mathbf{A}$ and fibers of $\mathbf{H}$. It will turn out that the embedding of $\mathbf{L}_{i}$ is determined entirely by data in the aa and ab tiles, and is independent of the bb tiles (which lie in the interior of $\mathbf{F}_{i}$ ). Since, by (10) and (11), the number of aa and ab tiles is independent of $i$, we will thus be able to conclude that even though the tiling of $\mathbf{F}_{i}$ is changing, the changes do not affect the boundary, so there cannot be infinitely many conjugacy classes of closed braids. 
With that plan in mind, let

$\tau=$ the number of singularities of types aa and $\mathbf{a b}$, i.e. $|\mathbf{a a}|+|\mathbf{a b}|$. $\omega=$ the number of points of $\mathbf{A} \cap \mathbf{F}_{i}$ which are vertices of these $\tau$ tiles.

$\rho=$ the number of endpoints of singular leaves which are on $\mathbf{L}_{i}$. Since each singular leaf on an aa (respectively ab) tile meets $\mathbf{L}_{i}$ twice (respectively once) the integer $\rho=2|\mathbf{a a}|+|\mathbf{a b}|$.

Let $s_{1}, \ldots, s_{\tau}$ be the singular points on the $\tau$ tiles of type aa and $\mathbf{a b}$, ordered to correspond to their cyclic order in the fibers of $\mathbf{H}$. That is, each $s_{k}$ is in $\mathbf{H}_{\theta_{k}} \cap \mathbf{F}_{i}$, where $0 \leq \theta_{1}<\theta_{2}<\cdots<\theta_{\tau} \leq 2 \pi$. Let $p_{1}, \ldots, p_{\omega}$ be the vertices of these $\tau$ tiles, ordered to correspond to their cyclic order on $\mathbf{A}$. Finally, let $\lambda_{1}, \ldots, \lambda_{\rho}$ be the points where the L-endpoints of the singular leaves in the tiling intersect the singular fibers of $\mathbf{H}$, ordered to correspond to their natural cyclic order on $\mathbf{L}_{i}$.

We now associate signs to the $s_{k}$ 's and $p_{j}$ 's. The sign $\xi_{k}$ of $s_{k}$ is + or - according as the sense of increasing $\theta$ agrees or disagrees with that of the outward-drawn normal to $\mathbf{F}_{i}$ at $s_{k}$. The sign $\delta_{j}$ of $p_{j}$ is + or - , according as the orientation of $\mathbf{A}$ agrees or disagrees with that of the outward-drawn normal to $\mathbf{F}_{i}$ at $p_{j}$.

Finally, we associate to the tiling of $\mathbf{F}_{i}$ a combinatorial symbol. Let $\mathbf{H}_{\theta_{k}}$ be a singular fiber containing the singularity $s_{k}$ on an aa tile, as depicted in the right column of Figure 3 . The singular leaves through $s_{k}$ have four endpoints, and these are alternately in the sets $\left\{p_{1}, \ldots, p_{\omega}\right\}$ and $\left\{\lambda_{1}, \ldots, \lambda_{\rho}\right\}$, so we can associate to $\mathbf{H}_{\theta_{k}}$ a cyclically ordered 4-tuple $\boldsymbol{4}_{k}=\xi_{k}\left(p_{1_{k}}, \lambda_{2_{k}}, p_{3_{k}}, \lambda_{4_{k}}\right)$. The sign $\xi_{k}$ is the sign of the singularity at $s_{k}$. The order of the 4 points is determined by their cyclic order on $\mathbf{H}_{\theta_{k}}$. In the case of an ab tile (again see Figure 3) three of the four endpoints of the singular leaves are in $\left\{p_{1}, \ldots, p_{\omega}\right\}$ and one is in $\left\{\lambda_{1}, \ldots, \lambda_{\rho}\right\}$. There is a signed and cyclically ordered 4-tuple $\mathbf{4}_{k}=\xi_{k}\left(p_{1_{k}}, p_{2_{k}}, p_{3_{k}}, \lambda_{4_{k}}\right)$ associated to the tile. Our combinatorial symbol is the array:

$$
\left((\tau, \omega, \rho),\left(s_{1}, \ldots, s_{\tau}\right),\left(\delta_{1}, \ldots, \delta_{\omega}\right),\left\{\lambda_{1}, \ldots, \lambda_{\rho}\right\},\left(\mathbf{4}_{1}, \ldots, \mathbf{4}_{\tau}\right)\right) \text {. }
$$

We now claim:

* The combinatorial symbol determines the conjugacy class of $\mathbf{L}_{i}$ as a closed braid, relative to the fibers of $\mathbf{H}$.

Parenthetical remark. The reader who is familiar with [B-M,I] will recognize similarities between the proof we are about to give and a related proof in $[\mathbf{B}-\mathbf{M}, \mathbf{I}]$; however there is a difference. In [B-M,I] we 
were interested in constructing $\mathbf{F}_{i}$ as an embedded surface, whereas our focus in this proof is entirely on $\partial \mathbf{F}_{i}$. We will see that the embedding of $\partial \mathbf{F}_{i}$ is completely determined by combinatorial data in the aa and ab tiles.

We return to the matter at hand. To prove $*$, we assume that we are given the combinatorial symbol, and proceed to construct $\mathbf{L}_{i}$ as a closed braid. We assume that the braid axis $\mathbf{A}$ is the $z$ axis in $R^{3}$, and that the fibers of $\mathbf{H}$ are the half-planes through $\mathbf{A}$. The combinatorial symbol gives us the integer $\omega$, and the first step is to choose $\omega$ points on $\mathbf{A}$ and label them $p_{1}, \ldots, p_{\omega}$, in order. Up to an isotopy of 3-space which preserves $\mathbf{A}$ it will not matter where we put them. The combinatorial symbol also tells us the integer $\tau$, so we choose $\tau$ fibers, selecting a point in the interior of each and labeling them $s_{1}, \ldots, s_{\tau}$, in the cyclic order in which the fibers occur. Passing to the $k$ th fiber, we may then construct the singular leaves in that fiber, as in the right column in Figure 3, with the help of the symbol $\mathbf{4}_{k}$. One of the singular leaves has both of its endpoints on $\mathbf{A}$, at $p_{1_{k}}$ and $p_{3_{k}}$ (in the notation of Figure 3 ). The combinatorial symbol $\mathbf{4}_{k}$ will tell us which points on $\mathbf{A}$ to use for $p_{1_{k}}$ and $p_{3_{k}}$. The other singular leaf crosses this one, and its two endpoints will be at $\lambda_{2_{k}}$ and $\lambda_{4_{k}}$ (if the tile is type aa) or at $p_{3_{k}}$ and $\lambda_{4_{k}}$ (if it is type ab). The points on $\mathbf{L}$ can be anywhere in the interior of $\mathbf{H}_{\theta}$ split along $p_{1_{k}} p_{3_{k}}$. Moving them will simply modify the embedding of $\mathbf{L}$ by an isotopy of $S^{3}$ which fixes $\mathbf{A}$ and each fiber of $\mathbf{H}$ setwise.

We have just described how to embed the singular leaves of the tiles which are adjacent to $\mathbf{L}$. The next task is to extend this embedding to a neighborhood of the singular leaves. The first thing to notice is that we know how to embed a neighborhood of $p_{1}, \ldots, p_{\omega}$ on $\mathbf{F}_{i}$. For, if we choose a neighborhood which is small enough it is radially foliated by its intersection with $\mathbf{H}$, so it must be a disc transverse to A. The sign $\delta_{j}$ of the point $p_{j}$ tells us the side of the surface which is pierced by $\mathbf{A}$. Next, notice that we can embed a little neighborhood of the singular leaves, for such a neighborhood is transverse to fibers everywhere except at the singular point, moreover the sign $\xi_{k}$ of the singularity tells us which side of $\mathbf{F}$ faces in the direction of increasing $\theta$ at the singular point. The last thing to do is to fill in regions on $\mathbf{F}$ which lie between adjacent points $\lambda_{i}$ and $\lambda_{i+1}$ on $\mathbf{L}$. See Figure 10 (on next page). These regions are foliated by radial arcs which emanate from the vertex, so they are everywhere transverse to the fibers of $\mathbf{H}$. The leaves of the foliation are level sets for the polar 


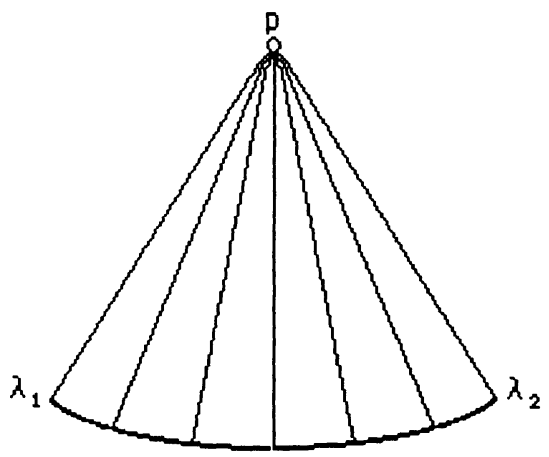

Figure 10

angle function, so they determine the embedding uniquely, up to an isotopy of $S^{3}$ which fixes each fiber setwise. When we are done we will have embedded a tiled neighborhood of the part of $\mathbf{F}_{i}$ which is closest to $\mathbf{L}_{i}$, and in particular we will have embedded $\mathbf{L}_{i}$ in $S^{3}$.

Recall that we have passed to a subsequence in which the changes which occur in the tiling of $\mathbf{F}_{i}$ as $i$ is increased are restricted to the interior of $\mathbf{F}_{i}$ and to vertices of type bbbb which are at the intersection of four type bb tiles. From this it follows that the integers $\rho, \omega$ and $\tau$ are independent of $i$. Since at most finitely many distinct combinatorial symbols are possible for fixed values of $\rho, \omega$ and $\tau$, and since the combinatorial symbol determines the embedding, we conclude that there are at most finitely many embeddings which are possible for $\mathbf{L}_{1}=\partial \mathbf{F}_{i}$. Thus only a finite number of conjugacy classes can occur among the links $\mathbf{L}_{i}$ in our sequence. Since this violates the hypotheses of Theorem 1, we conclude that this case does not occur.

The only thing we need to change if $\mathbf{L}_{i}$ has more than one component is the instructions for filling in the pie-shaped regions. Modify the combinatorial data by changing the labeling of the L-endpoints of the singular leaves to

$$
\left(\left(\lambda_{1,1}, \ldots, \lambda_{1, \rho_{1}}\right),\left(\lambda_{2,1}, \ldots, \lambda_{2, \rho_{2}}\right), \ldots,\left(\lambda_{t, 1}, \ldots, \lambda_{t, p_{t}}\right)\right),
$$

where the second subscript indicates the component. We may then proceed as before, one boundary component at a time, to fill in the regions next to the link.

If $\mathbf{F}$ is not connected, we proceed as before up to Lemma 5. We then replace Lemma 5 by the assertion that some component of $\mathbf{F}_{i}$ must have a tiling $\mathbf{F}_{i 1}$ in which the number of vertices grows without 


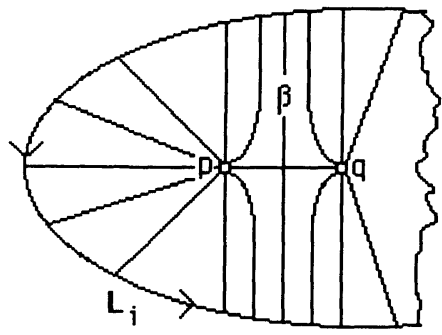

$p$ is a type a vertex

$\beta$ is a singular leaf in the foliation of $\mathbf{F}_{\mathbf{i}}$

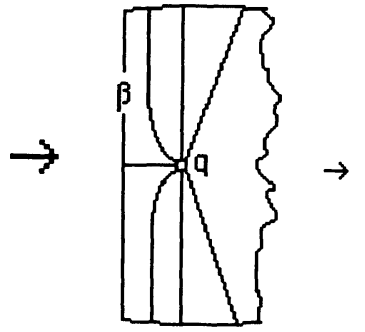

Delete the trivial loop bu cutting $\mathbf{F}_{\mathbf{i}}$ along $\beta$

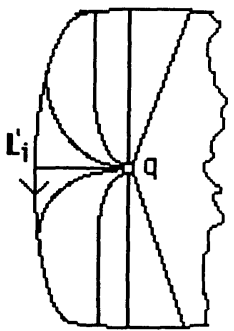

Perturb the cut surface to recover a closed braid

FIGURE 11

bound. In general, there will be, say, $t$ components. So, instead of a sequence of tiled surfaces $\left\{\mathbf{F}_{i} ; i=1,2,3, \ldots\right\}$ we will have tiled surfaces:

$$
\left\{\mathbf{F}_{i 1} \cup \mathbf{F}_{i 2} \cup \cdots \cup \mathbf{F}_{i t} ; i=1,2,3, \ldots\right\},
$$

where particular surfaces have tilings in which the number of vertices grows without bound. Some of these subsurfaces may have more than one boundary component, it will not matter. Lemma 5 applies to each subsurface. Lemmas 6 and 7 do too.

Thus we have proved: If our link $\mathscr{L}$ has infinitely many conjugacy classes of $n$-braid representatives, then all but finitely many of them admit exchange moves. This is a weak version of Theorem 1 .

To prove the stronger version, we must ask how the infinitely many conjugacy classes which admit exchange moves are related to one another. By our proof, each time that there is an exchange move, there is a vertex of type a or of type bb or ab with sign +-, or a vertex of type bbb. We first prove that we may assume there are no vertices of type a. Suppose, on the contrary, that there are infinitely many $\mathbf{F}_{i}$ 's which contain at least one vertex of type a. A vertex of type a always occurs on an "end tile," as in Figure 11. One of the singular leaves in that tile is a separating leaf $\beta$. If we cut $\mathbf{F}_{i}$ along $\beta$, and then modify the cut edge slightly so that the modified surface $\mathbf{F}_{i}^{\prime}$ is transverse to the foliation near the cut, the boundary of the modified surface will be a new closed braid $\mathbf{L}_{i}^{\prime}$ which is, in effect, obtained from $\mathbf{L}_{i}$ by deleting the trivial loop. Notice that the changes we just made do not alter the tiling in the complement of the end tile. So, after we delete our trivial loop we will obtain a new tiled surface $\mathbf{F}_{i}^{\prime}$ with all the properties of the old except: there is one less aa tile and 
the braid index has been reduced by 1 . This process may be repeated on $\mathbf{F}_{i}^{\prime}$ if the tiling of $\mathbf{F}_{i}^{\prime}$ contains a vertex of type a. However, it must end after $k \leq n$ such repetitions because each deletion reduces the braid index. Let $\left\{\mathbf{F}_{i}^{\prime \prime} ; i=1,2,3, \ldots\right\}$ be the infinite sequence of surfaces obtained after all type a vertices have been eliminated. Divide these into equivalence classes so that the braid index $n^{\prime \prime}$ is constant in each equivalence class. At least one of these equivalence classes must contain infinitely many members, because the original sequence did. Moreover, the surfaces in the new infinite sequence must have infinitely many distinct tilings, because we had infinitely many distinct tilings in our original sequence and all we did was to cut off finitely many end tiles from each $\mathbf{F}_{i}$. But then by our earlier work, we conclude that the tiling of infinitely many $\mathbf{F}_{i}$ 's in the original sequence contains a vertex of type bb or ab with sign +- or bbb.

Passing to a subsequence, we may assume that every $\mathbf{F}_{i}$ contains a vertex of type bb or ab with sign +- or bbb. By Lemma 4, each closed braid $\mathbf{L}_{i}=\partial \mathbf{F}_{i}$ admits a complexity-reducing exchange move. When we change an essential $\mathbf{b}$-arc to an inessential one we reduce the number of vertices in the tiling, and so reduce the complexity. For details see [B-M,IV and V]. The process of making exchange moves must therefore end.

Recall that when we began our work, we chose a spanning surface F for our link which had maximal Euler characteristic. The choice of $\mathbf{F}$ was not unique. The surfaces $\mathbf{F}_{i}$ which have been the object of our investigation here were then determined by finding homeomorphisms $h_{i}:\left(S^{3}, \mathbf{L}\right) \rightarrow\left(S^{3}, \mathbf{L}_{i}\right)$, and setting $\mathbf{F}_{i}=h_{i}(\mathbf{F})$. Later we introduced several types of modifications. Some of those modifications involved the passage to a subsequence. Others involved an isotopic deformation of $\mathbf{F}_{i}$. For example, when we changed $\mathbf{F}_{i}$ to a Markov surface, or when we cut off the end tiles, or when we modified $\mathbf{L}_{i}$ by an exchange move, we were changing $\mathbf{F}_{i}$ by isotopy. This means that each surface $\mathbf{F}_{i}^{\prime}$ in our final sequence is still homeomorphic to our fixed surface $\mathbf{F}$. If the set of complexities $\left(C\left(\mathbf{F}_{i}^{\prime}, \mathbf{H}\right) ; i=1,2,3, \ldots\right)$ is bounded, then by the main theorem in [B-M,I] there can be only finitely many distinct conjugacy classes among the links $\mathbf{L}_{i}^{\prime}=\partial \mathbf{F}_{i}^{\prime}$. If, on the other hand, the set of complexities is unbounded, there will be a contradiction to Lemmas 4, 5, 6, 7 of this paper. The proof of Theorem 1 is complete.

Proof of the Corollary. Notice that the exchange move as defined in Figure 1 does not change the exponent sum of an $n$-braid. Since, by 
the proof of Theorem 1, it is possible after repeated applications of exchange moves to produce one of the finitely many conjugacy classes of Theorem 2 of [B-M,I], we can only have finitely many possible exponent sums.

Proof of Theorem 2. The proof is the same as the proof of Theorem 1. Just notice that nowhere is it used that all the $\mathbf{L}_{i}$ 's represent the same link type. The only fact we needed was that all the $\mathbf{L}_{i}$ 's bound incompressible Seifert surfaces of fixed genus.

\section{REFERENCES}

[Be] D. Bennequin, Entrelacements et equations de Pfaff, Asterisque, 107-108 (1983), 87-161.

[B-M] J. Birman and W. Menasco, A Calculus on Links in $S^{3}$, to appear in the Proceedings of the International Conference on Knots, Osaka, Japan, August 14-18, 1990.

[B-M,I] _ Studying links via closed braids I: A finiteness theorem, Pacific J. Math., 154 (1992), 17-36.

[B-M,II] _ Studying links via closed braids II: On a theorem of Bennequin, Topology Appl., 40 (1991), 71-82.

[B-M,III] _ Studying links via closed braids III: Classifying links which are closed 3-braids, Pacific J. Math., to appear.

[B-M,IV] __, Studying links via closed braids IV: Split and composite links, Invent. Math., 102, Fasc. 1 (1990), 115-139.

[B-M,V] _ Studying links via closed braids V: The unlink, Trans. Amer. Math. Soc., 329 (1992), 585-606.

[J] V. R. F. Jones, Hecke algebra representations of braid groups and link polynomials, Annals of Math., 126 (1987), 335-388.

[VB] James Van Buskirk, Prime positive knots with inconjugate minimal string braid representatives, preprint, University of Oregon.

Received November 17, 1990 and in revised form July 15, 1991. Partially supported by NSF grants DMS-88-05672 and DMS-90-02673.

COLUMBIA UNIVERSITY

NEW YoRK, NY 10027

E-mail address: jb@math.columbia.edu

AND

SUNY AT BUFFaLo

BUfFALO, NY 14214

E-mail address: mthtwwm@ubvmsd.cc.buffalo.edu 



\title{
PACIFIC JOURNAL OF MATHEMATICS \\ Founded by \\ E. F. BeCKenbach (1906-1982) F. Wolf (1904-1989)
}

\section{EDITORS}

\author{
V. S. VARADARAJAN \\ (Managing Editor) \\ University of California \\ Los Angeles, CA 90024-1555 \\ vsv@math.ucla.edu \\ Herbert Clemens \\ University of Utah \\ Salt Lake City, UT 84112 \\ clemens@math.utah.edu \\ F. Michael Christ \\ University of California \\ Los Angeles, CA 90024-1555 \\ christ@math.ucla.edu \\ THOMAS ENRIGHT \\ University of California, San Diego \\ La Jolla, CA 92093 \\ tenright@ucsd.edu
}

\author{
Nicholas ERcolani \\ University of Arizona \\ Tucson, AZ 85721 \\ ercolani@math.arizona.edu \\ R. FINN \\ Stanford University \\ Stanford, CA 94305 \\ finn@gauss.stanford.edu \\ VAughan F. R. Jones \\ University of California \\ Berkeley, CA 94720 \\ vfr@math.berkeley.edu \\ STEVEN KeRCKHOFF \\ Stanford University \\ Stanford, CA 94305 \\ spk@gauss.stanford.edu
}

\author{
C. C. MOORE \\ University of California \\ Berkeley, CA 94720
}

MARTIN SCHARLEMANN

University of California

Santa Barbara, CA 93106

mgscharl@henri.ucsb.edu

\author{
HAROLD STARK \\ University of California, San Diego \\ La Jolla, CA 92093
}

\section{SUPPORTING INSTITUTIONS}

UNIVERSITY OF ARIZONA
UNIVERSITY OF BRITISH COLUMBIA
CALIFORNIA INSTITUTE OF TECHNOLOGY
UNIVERSITY OF CALIFORNIA
MONTANA STATE UNIVERSITY
UNIVERSITY OF NEVADA, RENO
NEW MEXICO STATE UNIVERSITY
OREGON STATE UNIVERSITY

\author{
UNIVERSITY OF OREGON \\ UNIVERSITY OF SOUTHERN CALIFORNIA \\ STANFORD UNIVERSITY \\ UNIVERSITY OF HAWAII \\ UNIVERSITY OF TOKYO \\ UNIVERSITY OF UTAH \\ WASHINGTON STATE UNIVERSITY \\ UNIVERSITY OF WASHINGTON
}

The Supporting Institutions listed above contribute to the cost of publication of this Journal, but they are not owners or publishers and have no responsibility for its content or policies.

\begin{abstract}
Mathematical papers intended for publication in the Pacific Journal of Mathematics should be in typed form or offset-reproduced (not dittoed), double spaced with large margins. Please do not use built up fractions in the text of the manuscript. However, you may use them in the displayed equations. Underline Greek letters in red, German in green, and script in blue. The first paragraph must be capable of being used separately as a synopsis of the entire paper. In particular it should contain no bibliographic references. Please propose a heading for the odd numbered pages of less than 35 characters. Manuscripts, in triplicate, may be sent to any one of the editors. Please classify according to the 1991 Mathematics Subject Classification scheme which can be found in the December index volumes of Mathematical Reviews. Supply name and address of author to whom proofs should be sent. All other communications should be addressed to the managing editor, or Elaine Barth, University of California, Los Angeles, California 90024-1555.

There are page-charges associated with articles appearing in the Pacific Journal of Mathematics. These charges are expected to be paid by the author's University, Government Agency or Company. If the author or authors do not have access to such Institutional support these charges are waived. Single authors will receive 50 free reprints; joint authors will receive a total of 100 free reprints. Additional copies may be obtained at cost in multiples of 50 .
\end{abstract}

The Pacific Journal of Mathematics (ISSN 0030-8730) is published monthly except for July and August. Regular subscription rate: $\$ 190.00$ a year (10 issues). Special rate: $\$ 95.00$ a year to individual members of supporting institutions.

Subscriptions, orders for numbers issued in the last three calendar years, and changes of address should be sent to Pacific Journal of Mathematics, P.O. Box 969, Carmel Valley, CA 93924, U.S.A. Old back numbers obtainable from Kraus Periodicals Co., Route 100, Millwood, NY 10546.

The Pacific Journal of Mathematics at P.O. Box 969, Carmel Valley, CA 93924 (ISSN 0030-8730) is published monthly except for July and August. Second-class postage paid at Carmel Valley, California 93924, and additional mailing offices. Postmaster: send address changes to Pacific Journal of Mathematics, P.O. Box 969, Carmel Valley, CA 93924.

\section{PUBLISHED BY PACIFIC JOURNAL OF MATHEMATICS, A NON-PROFIT CORPORATION} Copyright (C) 1992 by Pacific Journal of Mathematics 


\section{PACIFIC JOURNAL OF MATHEMATICS}

Volume $156 \quad$ No. $2 \quad$ December 1992

Surfaces in the 3-dimensional Lorentz-Minkowski space satisfying 201 $\Delta x=A x+B$

Luis Alías, Angel FerRandez and Pascual LuCAS

Lie algebras of type $D_{4}$ over number fields

209

BRUCE ALLISON

Subsemigroups of completely simple semigroups

251

Anne Antonippillai and Francis PAStiJn

Studying links via closed braids. VI. A nonfiniteness theorem

JOAN Birman and William W. MENASCO

Minimal orbits at infinity in homogeneous spaces of nonpositive curvature

MARÍA J. DRUETTA

Generalized horseshoe maps and inverse limits

SARAH ElizABETH Holte

Determinantal criteria for transversality of morphisms

DAN LAKSOV and ROBERT SPEISER

Four dodecahedral spaces

PETER LORIMER

Semifree actions on spheres

MONICA NiCOLAU

Conformal deformations preserving the Gauss map

ENALDO VERGASTA

Hecke eigenforms and representation numbers of arbitrary rank lattices 371

LYNNE WALLING 